6

\title{
Comparison and Analysis of Diffusion Models
}

\author{
Hannu Jaakkola \\ Tampere University of Technology, Information Technology (Pori) \\ P.O.Box 30, FIN-28601 Pori, Finland; E-mail: hj@pori.tut.fi
}

\begin{abstract}
A real diffusion process consists of a huge amount of interrelated variables. This complexity can be modelled by diffusion models building a simplified mathematical representation of the main features of the process as a time series of indicators describing the phenomenon in interest. Mathematical models are mostly used for technological forecasting purposes; forecasting is based on the best fit of the empirical data to the model formula and trend extrapolation outside the empirical period. The fit gives numeric values to the parameters of the model. Some of the models include also explanative factors - values of parameters describe behavioral properties of the process. The paper concentrates on mathematical diffusion models. The variety of models derived from the literature is introduced and analyzed. As an application of mathemat ical approach to the diffusion the diffusion of mobile phones is discussed. The focus of this paper is to point out problems in mathematical analysis and to discuss about alternative approaches.
\end{abstract}

\section{Keywords}

Diffusion models, diffusion process, technology management, mobile phones

\section{INTRODUCTION}

The diffusion of an innovation is a process, in which the innovation spreads through certain channels in the social system (target population) in time (Rogers 1983, p.5). According to Ayres (1969, p. xii) diffusion (of a new technology) is the evolutionary process of replacement of an old technology by a newer one for solving similar problems or accomplishing similar objectives. In the literature the term innovation appears typically in a context. By definition, innovation is the introduction or application of a new idea or invention; it is a change in the existing order. Essentially, diffusion or innovation concerns changing and managing a new technology by a model for predictive (or explanatory) purposes. An essential part of the model is presented by the diffusion effect, which has been defined by Mahajan et al. (1990, p. 38) as the cumulatively increasing degree of influence on an individual to adopt or reject an innovation.

Measuring the diffusion can be done either on qualitative or on quantitative level or combining these two approaches. Quantitative analysis is based on the time series of a selected variable or a group of variables representing the whole complicated process of the diffusion. 
Qualitative analysis is based on the methods crystallizing a united view from the collection of more or less intuitive standpoints on the diffusion. Martino (1993), in his book, has classified the following methods (for technological forecasting purposes):

- Delphi method (qualitative)

- forecasting by analogy (qualitative; semiquantitative)

- growth curves; figure of merit (quantitative; proportional to trend analysis)

- trend analysis and extrapolation (quantitative)

This paper concentrates on quantitative modelling of the diffusion.

A real diffusion process consists of a huge amount of variables and interrelations between them. This complexity can be modelled and managed by diffusion models. The primary purpose of diffusion models is to manage the regularity encountered in the process. Traditionally the model is a simplified mathematical representation of the main features of the process as a time series of indicators describing the phenomenon in interest. Mathematical models are mostly used for technological forecasting purposes; forecasting is based on the best fit of the empirical data to the model formula and trend extrapolation outside the empirical period. The fit gives numeric values to the parameters of the model. Some of the models also include explanative factors - values of parameters describe behavioral properties of the process.

An optional approach called heuristic (loose) modelling is derived from the principles of mathematical diffusion models and introduced by the author of this paper in several publications (Jaakkola 1994;1994a; 1993; 1992; 1992a; 1991; 1991a). The heuristic modelling means the intuitive approach to the analysis of the complex process. All the knowledge the analyst has on the process is used first to understand the changes of the process in the past and then to reason the future improvement. The principles used in the analysis are collected in the heuristic diffusion model, which is a generic model (collection of analysis principles) supporting the feature analysis.

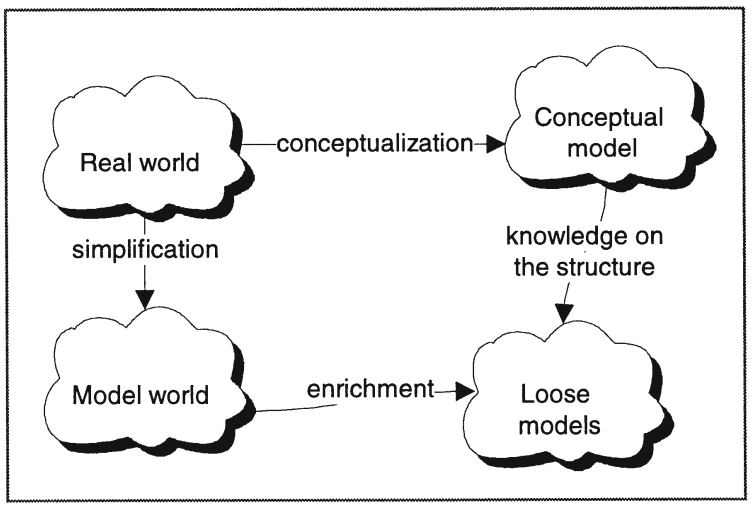

Figure 1 Modelling the diffusion process

This paper concentrates on mathematical diffusion models. The models are tools for diffusion analysis. Using the models in the right way consists of two factors: understanding the modelling principles and understanding the assumptions behind the model. Chapter 2 concentrates in the basic principles of the diffusion process. The variety of models derived from the literature is introduced and analyzed in chapters 3 and 4. As an application of mathemathical analysis the diffusion of mobile phones is studied in chapter 5 . The main result of this paper is the critical analysis of the properties of mathematical models used in diffusion analysis. The study will argument the need of improved mathematical methods and/or loose and a more heu- 
ristic approach in analysis to improve the usability of the models in practical problems (Figure 1); the simplified world of mathematical models requires structural knowledge on the real world. The special problems in connection with the analysis of IT diffusion are discussed. This critique is included in the conclusions in chapter 6.

The paper is one of a series of papers discussing the principles of mathematical diffusion models. In the paper (Jaakkola 1995) the general principles of mathematical models and their scope are discussed. The paper (Jaakkola 1995a) concentrates on the flexibility features of mathematical diffusion models.

\section{HISTORY AND THE ERAS OF DIFFUSION MODELS}

In the literature, the theory of diffusion models has its roots in the 19th century: a sociologist, Gabriel Tarde (1890) applied mathematical models in the spread of political ideologies. He noticed the power of imitation as the explanation factor in the diffusion. Since the beginning of 20 th century there exists a wide variety of models of the same kind applied in different fields, e.g. epidemiology, sociology, marketing, learning, anthropology, biology, technology diffusion and substitution; more about the history of modelling see e.g. (Jaakkola 1993a; 1993b).

The active period of technology diffusion research starts in the 1960's. The period from the beginning of the 1960's until now can be divided into three eras:

- the era of basic models (1960's and the beginning of the 1970's)

- the era of expanded models (1970's and the beginning of the 1980's)

- the era of new applications (from the 1980's)

In the era of basic models the research concentrated on the development of mathematical basic models. The models were simple mathematical formulas (statistical distributions) used to fit in the empirical time series data. The main thing in the use of models was forecasting by extrapolation: the parameters of the model were estimated by regression analysis methods.

In the era of expanded models the research work concentrated on the shortages of basic models: the fixed structure, inability to take into account the changes in the environment and inability to handle competitive innovations. A basic method was to add the flexibility of models by joining together two or more basic models. This kind of modifications focused in the dimensions of the changing environment, competitive innovations, multi-phase adoption, interaction between innovations etc. The main purpose in the use of models was still the forecasting by extrapolation. Most of "umbrella models" are created in this era.

The era of new applications has expanded the scope and flexibility of models. Typical new features are unbundling of the population of potential users, critical analysis of the adoption decision, improved capability to take into account the effect of environmental factors and the use of feedback mechanisms to adapt the behavior of the models to fit better in the behavior of the real world phenomena. The most meaningful is however the transition from the forecasting towards more complicated use of models:

- descriptive use: testing hypothesis to get information on the effect of different operations in the diffusion process

- normative use: the use of models to optimize the estimates of factors affecting in the diffusion process

In the same time period the complexity of models has grown and they have come nearer to simulation models than the models of former eras.

This paper concentrates on the analysis of the models of two first eras; the purpose of this paper is to analyze the properties of mathematical diffusion models to become acquainted with the functional power of typical forecasting models. The complicated "simulation type" feedback models do not include essential new information from that point of view. 


\section{BASIC MODELS}

\subsection{The Use of models}

\section{General model and the pressure function}

The diffusion process is typically modelled by using components described in Figure 2. Symbols in the figure denote the following:

$\mathrm{f}(\mathrm{t})$ non-cumulative adoption function; diffusion function

$\mathrm{F}(\mathrm{t})$ cumulative adoption function; adopter (distribution) function

$\overline{\mathrm{F}}$ potential adopter population (most of the models presuppose a fixed potential of adopters during the adoption period

$\{1\}$ the whole population in which the potential adopter population is a subset

$\{2\}$ the lower threshold level of penetration; if a substitution has progressed to this level it will proceed to its completion; practically, the threshold level is $10 \%$ of the potential, and the behavior of the diffusion process before this point is irregular (Fisher and Pry, 1972)

\{3\} on the inflection point $t=t^{*}, \mathrm{f}(\mathrm{t})$ has its maximum value and the first degree derivative of $\mathrm{F}(\mathrm{t})$ will change from positive to negative

\{4\} the upper threshold level, maturation level; after reaching this level of penetration, the process is practically finished and the behavior of the rest of the population is not regular and exactly modellable

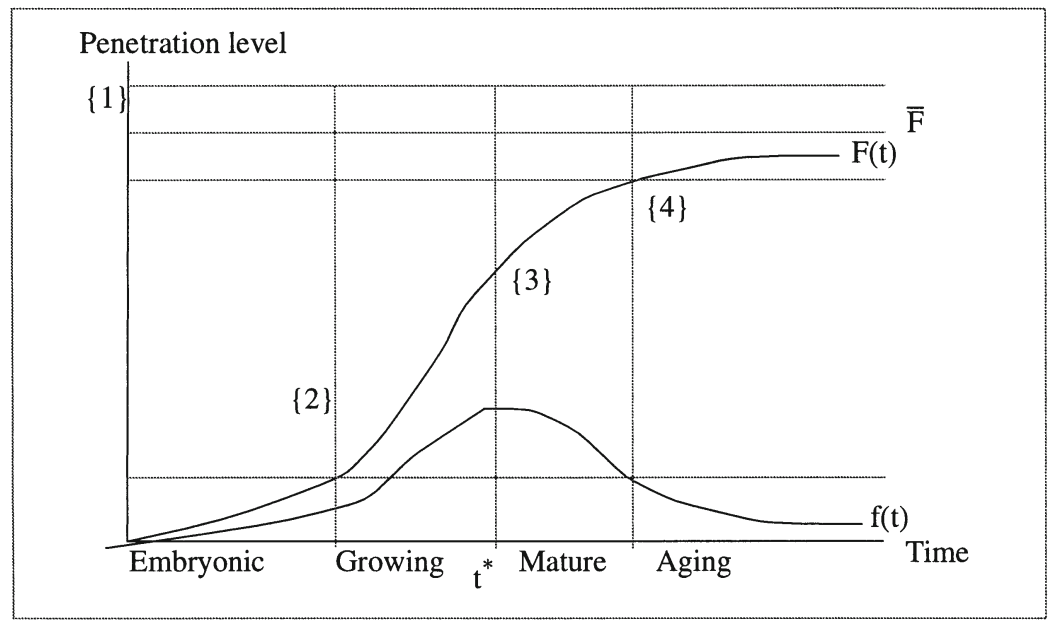

Figure 2 The components of diffusion models

The fast growth phase between points $\{2\}$ and $\{4\}$ is the focal point of most diffusion models. Some of the models are valid only in this area because of the irregular behavior in the beginning and at the end of diffusion, or because they are limited only to some part of the process (e.g. the models presuppose a symmetry relative to the inflection point and they describe only the area before or after that point). The whole period of diffusion can be divided into four phases with characteristic features of their own. These features are discussed in detail by Jaakkola 1993; 1993a).

A general form (see e.g. Majahan 1977; 1985), of the diffusion process can be presented 
according to the following formula:

$$
f(t)=a(t)(\bar{F}-F(t))
$$

in which $\mathrm{a}(\mathrm{t})$ is called the pressure function. The growth is proportional to the remaining user potential. The pressure function of a diffusion model describes the factor affecting the growth in the use of the innovation analyses. The pressure function $a(t)$ characterizes the structure of the diffusion model and it is defined:

$$
a(t)=\frac{f(t)}{\bar{F}-F(t)}
$$

The pressure function specifies the behavior of the model in the following way:

- internal model: the internal interaction (imitation) of the user potential directs the adoption; the value of $\mathrm{a}(\mathrm{t})$ approaches the value zero in the early beginning of the adoption process

- external model: adoption process is controlled by the external effect only (innovation effect); the value of $a(t)$ approaches a positive constant in the early beginning of the process

- mixed model: the diffusion process is a sum of internal and external effects

The growing value of the pressure function means a growing interest in the adoption of the product, and descending value a reduced interest.

\begin{tabular}{|c|c|c|c|c|}
\hline Model & Pressure function $a(t)$ & $\lim a(t)$ & $\begin{array}{l}\text { Analytic form of } \\
a(t)\end{array}$ & Class \\
\hline Logistic distr. & $b F(t)$ & 0 & Growing & I \\
\hline Gompertz distr. & $\frac{-b F(t) \ln F(t)}{1-F(t)}$ & 0 & growing & I \\
\hline Floyd & $b F(t)(1-F(t))$ & 0 & inverted $\mathrm{U}$ & I \\
\hline Sharif-Kabir & $\frac{b F(t)(1-F(t)}{(1-(1-\sigma) F(t)}$ & 0 & $\begin{array}{l}\sigma=1 \text { :inverted } U \\
\sigma<>1 \text { : growing }\end{array}$ & I \\
\hline v.Bertalanffy & $\frac{b F^{\theta}(t)\left(1-F^{1-\theta}(t)\right.}{(1-q)(1-F(t))}$ & 0 & $\begin{array}{l}\theta<1 \text { : growing } \\
\theta \geq 1 \text { : decreasing }\end{array}$ & I \\
\hline NSRL & $b F(t)^{\alpha}$ & 0 & $\begin{array}{l}\alpha=0 \text { : constant } \\
\alpha<>0 \text { : growing }\end{array}$ & I \\
\hline Bass & $\mathrm{p}+\mathrm{qF}(\mathrm{t})$ & $\mathrm{p}$ & growing & M \\
\hline NUI & $\mathrm{a}+\mathrm{bF}(\mathrm{t})^{\alpha}$ & $\mathrm{a}$ & $\begin{array}{l}\alpha=0 \text { : constant } \\
\alpha<>0 \text { : growing }\end{array}$ & M \\
\hline Exp distr. & $\mathrm{a}$ & $\mathrm{a}$ & Constant & $\mathrm{E}$ \\
\hline Weibull distr. & $\mathrm{kpt}^{\mathrm{k}-1}$ & $\begin{array}{l}\mathrm{k}>1: 0 \\
\mathrm{k}<1: \text { inf. }\end{array}$ & $\begin{array}{l}\mathrm{k}>1 \text { : growing } \\
\mathrm{k}<1 \text { : decreasing } \\
\mathrm{k}=1 \text { : constant }\end{array}$ & $\begin{array}{l}\mathrm{k}>1: \mathrm{I} \\
\mathrm{k} \leq 1: \mathrm{E}\end{array}$ \\
\hline Normal distr. & - & 0 & growing & I \\
\hline
\end{tabular}

Table 1 Properties of some diffusion models 
Pressure function is an optional way to introduce the analytic form of the model; the cumulative and non-cumulative diffusion functions can be derived from the pressure function representation solving the differential equation. Pressure functions of some diffusion functions described later in this paper are introduced in Table 1 (Jaakkola 1991, p. 83), indicating to the analyst the driving force of the adoption decision of the adopters.

\section{Analytic form of the models}

A typical use of the model is to fit the predefined mathematical function in the empirical time series data describing the spread of the product or the innovation; the data is used to estimate the parameters of the predefined mathematical models (either cumulative or non-cumulative diffusion function; best fit is based on simple regression analysis, e.g. least square sum). The parameters in the model are the parameters defined in the pressure function and the expected potential user population $\overline{\mathrm{F}}$. The predefined mathematical functions (distributions) used in models are e.g.

- logistic distribution (Blackman, Fisher-Pry, Dodd)

- derivatives of the logistic distribution (Floyd, Sharif-Kabir, Mansfield, Bass, Easingwood, Sharif-Ramanathan)

- flexible logistic (FLOG) distribution

- Gompertz distribution

- exponential distribution (Coleman, Fourt-Woodlock)

- normal distribution (Stapleton)

- lognormal distribution

- v. Bertalanffy distribution

- Weibull distribution (Sharif-Islam)

A common feature for the distributions is the S-shaped cumulative and bell-shaped non-cumulative adoption curve; the curves having a different shape (exponential, decayed exponential etc.) actually have a head in the beginning or a tail at the end out of the modelling period (Jaakkola 1995). The distributions differ from each other regarding the symmetry, the location of the inflection point and the amount of parameters. Both logistic and normal distributions are symmetric regarding the inflection point; Gompertz distribution is not symmetric but it has a fixed inflection point depending on the user potential $(\overline{\mathrm{F}})$; Weibull distribution has the inflection point depending on the parameter. A detailed analysis of mathematical properties of the distributions is not worth doing in this paper. Details are given by Jaakkola (1991, pp. 243252), Kumar\&Kumar (1992, p. 162) and Majahan et al. (1990, p. 12).

The reliability of the modelling differs in models and it is based on the length of the time series of empirical data regarding the basic assumptions of the model. The areas outside the fast growth phase are usually unstable and left out of the analysis in most models. There are also presumptions of reaching a certain penetration level to allow the use of models for forecasting purposes. These features will cause problems especially in the case of fast changing technologies, which diffusion typically can be described as a series of exponential growth curves; the analyst's interest in the older generation curves decreases after the appearance of the newer one. The most serious problem in the use of mathematical models is the need to fix the values of model parameters in the beginning of the analysis: the result model is as good as the parameter "guess".

\subsection{Some basic models}

\section{Fisher-Pry model}

This model is perhaps one of the most known diffusion models. It is based on the idea of using Pearl's formula (epidemic model) to describe the diffusion process. This model is a derivative 
of the logistic model and introduced by Raymond Pearl (1925). The epidemic model was applied by Fisher and Pry (1972) in the replacement of the old technology by the new one. The model is based on the following expectations:

- when the diffusion has reached a certain penetration level (lower threshold), the process will progress until full penetration

- the lower threshold level is $0.1 * \overline{\mathrm{F}}$ and the upper one $0.9 * \overline{\mathrm{F}}$; the process is regular between these points and symmetric regarding the inflection point in the middle (time when the penetration $0.5 * \overline{\mathrm{F}}$ is reached) of the process

- the proportional growth of the user population is constant regarding the remaining potential until the inflection point; the user potential is expected to be fixed (not changing as a function of time)

The model is based on the imitation effect: the adoption decision is based on the experiences of the earlier adopters. Because of that there is the expectation that the diffusion process has its beginning in the "spaceless past" which guarantees the reaching of the lower treshold level and symmetrically it continues in the "spaceless future" reaching the full penetration somewhere after the time where the upper threshold level is reached.

\section{Blackman and Mansfield}

The Blackman model (1972) is a derivative of Mansfield's model (1961). The interrelation between these models also describes the interrelation between two typical uses of the models: the use to describe the diffusion of a product (Mansfield) and the use to describe the changes in the performance of the product (figure of merit; Blackman). In the Blackman model the changes in the adoption depend on the

- ratio $\mathrm{F}(\mathrm{t}) / \overline{\mathrm{F}}$ (the proportion of the users in the potential amount of users); the user potential is expected to be fixed

- the expected benefit that can be achieved

- the magnitude of the investment

The adoption function is based on the Taylor polynome introduced by Mansfield: the function is built by combining all three parameters in the form of Taylor transformation. Blackman expects that the environment of the diffusion is unchanged in the diffusion period; this assumption simplifies the function to be a derivative of the logistic distribution and gives semantics to the parameters of the pressure function. The values of the parameters describe the power of the factors mentioned above to the process and are useful e.g. in comparing the similarity of two different diffusion processes (forecasting by analogy). By the characteristics the model belongs to the class of imitation models and it is symmetric regarding to the inflection point $(0.5 * \overline{\mathrm{F}})$. In his own evaluations Blackman has noticed that the biggest shortcoming in the model is the assumption of the static environment; it will cause too optimistic short run and too pessimistic long run forecasts in the use of the model.

\section{Bass' first purchase model}

Bass' model (1969) is one of the oldest and most modified (see e.g. Majahan 1977; 1990) diffusion models. The original application of the model is the forecast of the first purchase of consumer durables; the effect of new generations and multiple purchases is eliminated by shortening the analysis period. The model is improved to take into account the interaction between product generations by Norton and Bass (1987).

The pressure function in Bass' model has two parameters: the other one describes the innovator effect and the other one the imitation effect in the diffusion decision. The structure of the potential user population is analyzed by Rogers (1970) in his well-known book "Diffusion of Innovations" and further developed by Majahan e.g. in papers (1990; 1990a).The imitation 
effect describes the amount of adopters deciding the adoption independently (internal pressure). The rest of the user potential is controlled by external pressure - imitating the earlier adopters (innovators and imitators already made the positive adoption decision).

The Bass' model has following properties:

- the model is asymmetric and the inflection point (varies in the area from 0 to $0.5 * \overline{\mathrm{F}}$ ) depends on the parameter values

- in the analysis, the model assumes the availability of empirical data exceeding the inflection point (compare e.g. Fisher-Pry assumes exceeding the lower treshold only)

The model assumes static environment of the diffusion and is useful for first-purchase analysis only; it can be used in the empirical data reaching the inflection point.

\section{ADDING FLEXIBILITY}

The critique against the usefulness of basic diffusion models concerns on the factors causing inflexibility in models. The inflexibility is caused by the fixed analytic form of the model: in forecasting the use of the mathematical model is based on the "best fit" evaluation of parameters of the model and the inflexibility will cause the growth of error factor in the fit process. An complementary approach to the development of diffusion models is based on the concept of "umbrella models". An umbrella model joins the properties of two or more basic models having different behavior and is based on the assumption that the characteristics of the diffusion process are changing in the run of the diffusion. This approach will

- increase flexibility (and leads to better fit than fixed models)

- add new (non explanatory) factors to the model

Two examples of the dedicated umbrella models are the Sharif-Kabir model (1976) and SharifRamanathan model (1981).

\section{Sharif-Kabir Model}

Sharif-Kabir model is the technology replacement model, in which three models having different behavior are combined together. The basic models are

- Blackman model

- Fisher-Pry model

- Floyd's model (1968) for non-competing technologies

The two first models were introduced earlier in this paper. Floyd's model is nonsymmetric (inflection point $0.333 * \overline{\mathrm{F}}$ ) derivative of the logistic distribution. According to Sharif and Kabir, the two first models lead typically in too optimistic and the last one in too pessimistic forecasts. Combining the models results a generalized model, that is more reliable than any of the component models alone.

The combination of models is created by joining the two first models (Fisher-Pry model can be interpreted to be a special case of Mansfield model) and combining this with Floyd's model by using a coefficient (value $0 . .1$, specifying the power of the component models in the combined result model). The values of the delay coefficient describe the delay of the adoption compared to the most optimistic estimate (logistic distribution). The value of the delay coefficient depends on the

- data scattering (DS)

- data extent (DE; the time span of the empirical data in reflect to the time span of the analysis)

- the last value of $\mathrm{F}(\mathrm{t})$ in empirical data $(\mathrm{fl})$

- the effective life span (the time from the penetration level from $0.1 * \overline{\mathrm{F}}$ to $0.9 * \overline{\mathrm{F}}$

In the early diffusion DS and $\mathrm{fl}$ are dominating the value of the delay coefficient. The scattered empirical data increases error level; the abundant portion of the empirical data under the lower threshold level $(10 \%)$ causes pessimistic forecasts. The life time of the innovation also has an 
effect on the value of the coefficient: the shorter is the life time of the innovation the smaller its effect in the coefficient. The length of the time series of the empirical data increases the value of the coefficient. The flexibility of the model is illustrated in Figure 3.

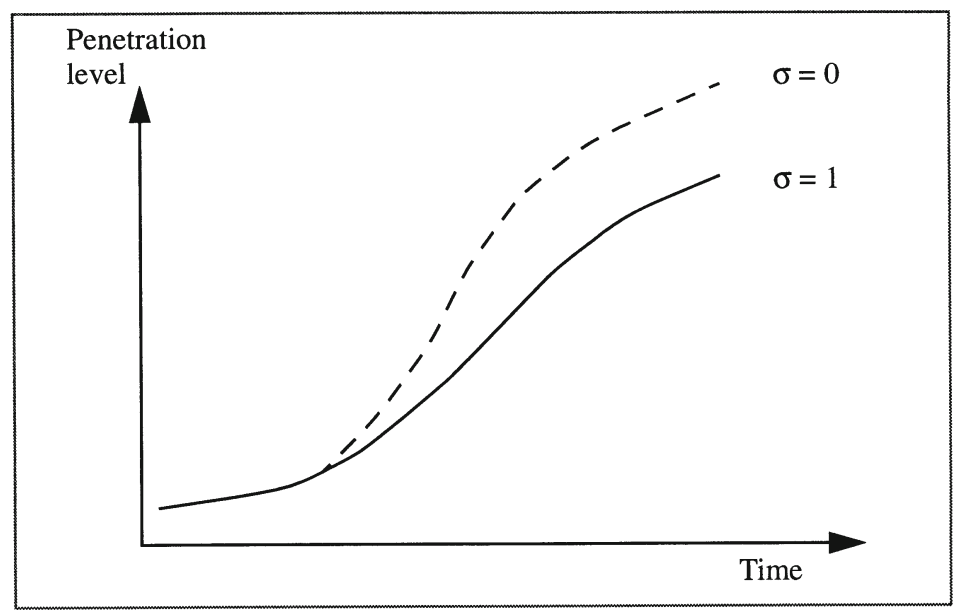

Figure 3 The effect of the delay factor in Sharif-Kabir model (Jaakkola 1991, p. 70)

\section{Sharif Ramanathan Model}

Sharif and Kabir combined the models havingthe same kind of basic properties but different behavior in different parts of the diffusion process. In his newer study Sharif has used an alternative approach by joining two component models having different behavior. Sharif and Ramanathan have combined Coleman's model (1964) and Dodd's model (1953) which both belong to the early history of the diffusion studies. Coleman's model is an innovator model (the amount of new users in time $t$ is independent of the amount of existing users $F(t)$. The analytic form of the diffusion function is reversed exponential function and the adopter distribution a decayed exponential function describing the fast decrease of the external pressure in the adoption decision. The assumptions of the model are:

- fixed user potential

- full penetration will be reached during the diffusion period

- the source of innovation is fixed it effects all adopters in the same way

In the original article Coleman has a long discussion on the interpretation of the parameters of the functions (these interpretations are not in the development of Sharif-Ramanathan model)

In Dodd's model the diffusion function is a derivation of the logistic distribution and the assumptions in the model are the same as above. The arguments of the adoption decision are expected to be same during the whole diffusion period and the their effect in the potential user are expected to be homogenous in the whole population.

The purpose of Sharif and Ramanathan has been to create a model having both exponential and logistic behavior built in the model. The model is an innovator-imitator model having Sshaped diffusion function; its flexibility covers Blackman's, Bass' and Fourt-Woodlock (1960) model. The flexibility of the model is described in Figure 4.

Coleman's exponential distribution corresponds very well to the diffusion of fashion products; the adoption is very fast and the adoption period short. Dodd's model and logistic distribution is better suited to typical phenomena. Changing the parameters of the SharifRamanathan model the behavior of the model can be varied inside these two ends. 


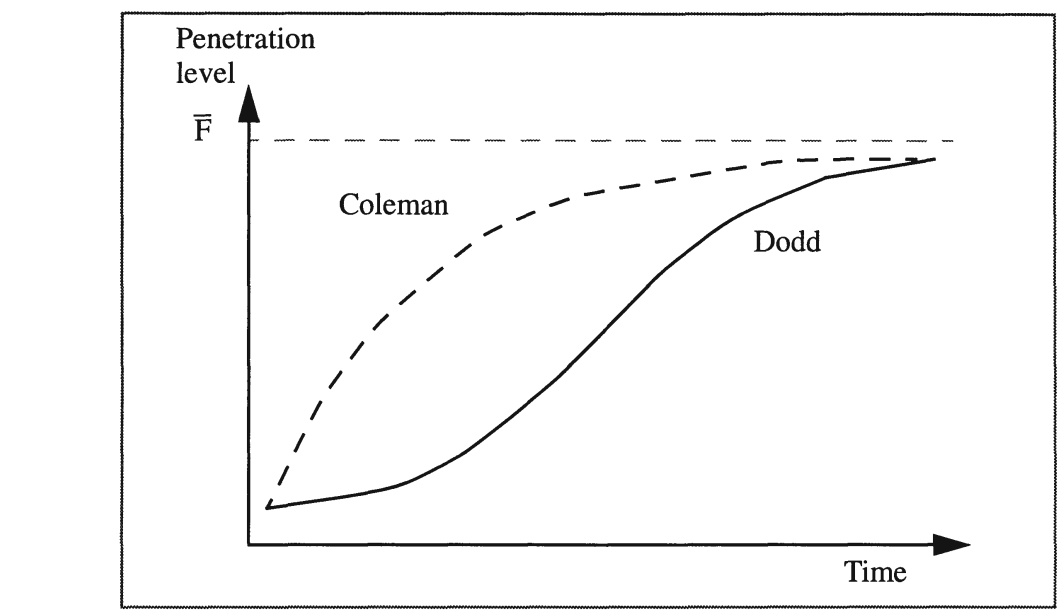

Figure 4 The flexibility area in Sharif-Ramanathan model

\section{More advanced flexibility properties}

The flexibility of the models described above are implemented by adding characteristics of two different basic functions in one model and varying the value of the coefficient describing the importance of the components. The flexibility in general is a concept including different means:

- a flexible model: varying inflection point and symmetry/asymmetry

- a dynamic model: values of parameters (user potential, imitation factor, the structure of the user potential, etc.), can be varied during the diffusion period as a function of time (or some other argument)

- an adaptive model: the model adapts in the empirical data (feedback property; e.g. error factor of the regression adapts the model)

Flexibility of the models is achieved by the selection of distributions having built in flexibility needed for the analysis. Such distributions are e.g. Gompertz distribution (changing inflection point) and Weibull distribution (changing inflection point and symmetry). The adaption is a technical solution to fit the model pattern in the empirical data. Dynamic features in the model are implemented by replacing the constant parameters by the functions depending on some variables controlling the process.

The models developed to implement high flexibility are e.g. NSRL (Non Symmetric Responding Logistic Model) introduced by Easingwood et al. (1981), NUI (Non Uniform Influence) introduced by Easingwood et al. (1983) and General Rationalized Models (GRM) by Skiadas (1985). A detailed analysis of these models is done by Jaakkola (1995a); the paper concentrates on the flexibility properties of diffusion models. The main finding in the paper is the parallel increasing flexibility and inability to explain the process features.

The problem of the increasing flexibility in diffusion models is the conflict between the needs to model the real phenomenon and to create flexibility. In principle, the high level of flexibility makes possible for a good fit of the model in the empirical data, but at the same time it obscures the understandability of the behavior of the model and decreases the opportunity to use the parameters of the model to interpret the properties of the process or in the comparison of two distinct processes. The flexibility becomes the end in itself and the primary purpose of the model remains in the background. The variety of the models developed for flexibility purposes are not handled here in detail. 


\section{DIFFUSION OF IT}

\section{About IT Diffusion Studies}

A wide variety of diffusion studies are introduced in literature. Jaakkola (1991, pp. 113-122) analyses ten empirical studies. In the same book a deep analysis of the diffusion of IT in Finnish industry is introduced (see also Jaakkola 1992b; 1993b). An interesting review of IT diffusion studies is given by Fichman (1992); the study includes an overview of 36 research papers and articles. A wide spectrum of like studies can be found in literature.

The diffusion of IT can be measured in several different ways. Indicators used in diffusion studies are e.g.

- the amount of IT users among the employed people, time series based on that data and international comparisons; these indicators describe the level of utilization of new technology in different sectors of the society

- the relative amount of computers at homes; these studies are implemented by market research companies and results indicate the spread of computer culture as a part of daily life

- the utilization of IT in different sectors of the industry and business; these indicators describe the progress in the different user segments and application sectors of the society

- the structure of the computer base and the changes in the annual sales; these kinds of studies are implemented by market research companies and describe the technical improvements as a quality dimension of the use of IT

- the utilization level of a product indicating the process in the researcher's interest In most cases there are two main problems causing uncertainty in the results of analysis: (1) rapid changes and (2) short time series. Rapid changes are realized in the analysis as the sequence of product generations meaning the replacement of the old generation product by the new one before the penetration reaches the level of reliable analysis; short time series are the consequences of rapid changes and do not allow the use of exact mathematical analysis, which typically expect the break-trough point to be reached to get the model reliable enough for forecasting purposes. The need of modelling comes from the need to manage the changes - predict the demand of products, predict the behavior of future generations of a product in markets etc. Understanding the power (and the lack) of mathematical diffusion models provides valuable information for the development of the subjective (heuristic) application specific combined methods for the bases of the change management.

The use of mathematical models follows the steps below

- decision cocerning the break point between empirical data and forecast (mostly all empirical data available is used); this decision means that the static environment of the break point is accepted to be the environment of the extrapolation period

- specificying the parameter values (e.g. user potential) not evaluable in the "best fit" phase (a new fixed value in the static environment)

- solving the values of model parameters (fitting the model to the empirical data; best fit gives values to the parameters; adding new values to the static environment)

- extrapolation (forecast) in the static environment

As an example of this process the diffusion of mobile phones is analyzed below.

\section{An example of the mathematical analysis}

An example of mathematical analysis and an introduction to the problems included in the analysis is given below. The empirical data analysed is the number of mobile telephone licenses in Finland. Mansfield's model (see chapter 3 ) is used in the analysis:

$$
F(t)=\frac{e^{c_{1}+c_{2} t}}{1+e^{c_{1}+c_{2} t}} \bar{F}
$$




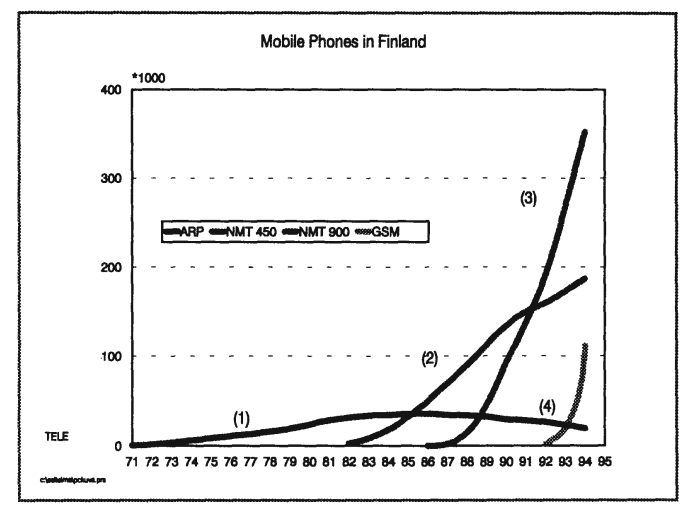

Figure 5 Mobile phone diffusion in Finland

Mobile phone diffusion in Finland is an interested application for analysis for several reasons:

- the history of mobile phones in Finland includes four generations following each other allowing generation based analysis in addition to the analyses of the total phenomenon (Figure 5); manual (today semi automatic) ARP network (1) started to operate in 1980 has 19.000 licences (highest 35.000 in 1986), $450 \mathrm{MHz}$ analogic network NMT-450 (2) started to operate in 1982 has 187.000 licences, $900 \mathrm{MHz}$ analogic network NMT-900 (3) started to operate in 1986 has 352.000 licences and two competing digital GSM networks (4) started to operate in 1992 have 110.000 licenses; the growth rate in NMT-450 is 8\%, NMT 900 has the rate of $30 \%$ and GSM hundreds of percents

- Finland is the leading country in the adoption of mobile phones, which means that the experiences in Finland can be used in the plans concerning less penetrated countries; the penetration rate in August 1995 is $18 \%$ (900.000 licenses in the population of 5 Million), which has almost reached the level of the EUTELIS (1993) forecast by the year 2010 in Europe (penetration rate $30-50 \%$ in business user and $15-35$ in private user segment); the most positive Finnish forecasts expect the penetration level of $100 \%$ of the population to be reached by the beginning of next decade

- there seems to be a trend towards private sector dominance in the new mobile phone licences; today almost $60 \%$ of new licences are registered for private people and this share is increasing all the time; the share of the private sector seems to grow several percents in a year; there is a good reason to believe that in the static situation the share $90 \%$ private, $10 \%$ business will be reached in the beginning of next decade (i.e. the static situation in fixed line telephones today)

There are some other indicators but the number of licenses that can be used in empirical analysis, e.g. the number of calls in mobile telephone network. According to Telecommunications Statistics (1995, p. 38) the number of calls in whole network was 292.8 Million (12.5\% in digital networks and $87.5 \%$ in analogic ones). The forecast is that the number of calls in two digital networks is increasing from 36.7 Million in 1994 to 100 Million in 1995.

The behavior of the four diffusion processes includes a huge number of explanative factors not modellable in the model: changing user potential, network coverage, network capacity problems, pricing of the licenses, pricing of the calls and monthly fee, services etc. Short time series make impossible the mathematical analysis of individual processes, which seem to fill the properties of the theory of competing and replacing technology generations. The purpose 
of the following example is to show the difficulties rising up in practical analysis of fast changing technologies. Mansfield's model is selected; the model used could be some of the other ones introduced earlier in this paper.

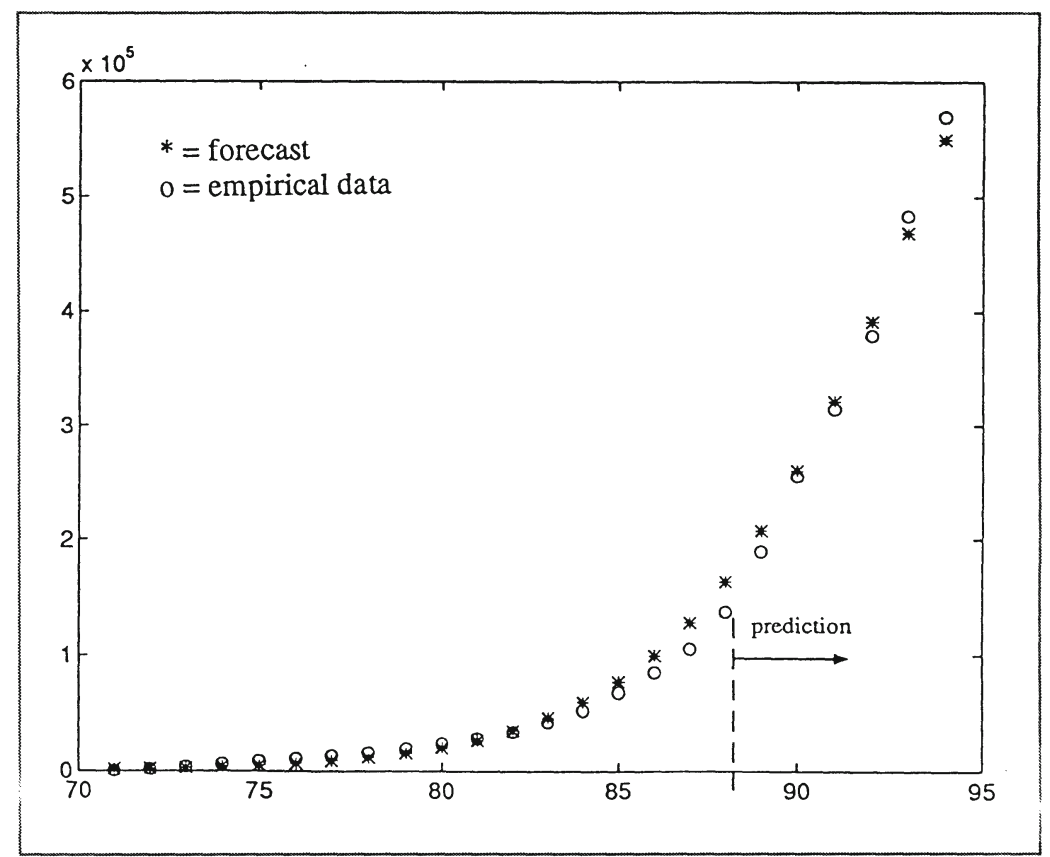

Figure 6 Mobile phone diffusion - empirical data 1971.. 1988, forecast 1989 .. 1994

The Analysis on mobile phone data is done in two phases:

1. sensitivity on empirical data was tested by moving the break point of empirical data and forecast in the area 1985. 1988; the rest of the curve was built by forecast, which was compared to the real empirical data in the period from break point to 1994

2. sensitivity on user potential was tested and forecasts based on different expectations on user potential were produced

Table 2 Parameter values in the break point analysis $(\overline{\mathrm{F}}=1.2$ Million)

\begin{tabular}{|l|l|l|}
\hline Year & $\mathrm{C}_{1}$ & $\mathrm{C}_{2}$ \\
\hline \hline 1985 & -6.97 & 0.30 \\
\hline 1986 & -6.92 & 0.29 \\
\hline 1987 & -6.89 & 0.28 \\
\hline 1988 & -6.86 & 0.28 \\
\hline
\end{tabular}

Sensitivity on empirical data was tested by moving the break point between empirical data and forecast in the area from 1985 to 1988; the purpose of the test was to find the minimum proportion of the empirical data needed to produce reliable forecasts (in the existing empirical data). The change of values of model parameters is represented in Table 2. In the analysis, a 
short empirical data period seems to produce too optimistic forecasts and the break point in 1988 seemed to produce stability in the model (Figure 6). In the analysis the number of licenses in 1988 was 105.860 which represents the share of $8.8 \%$ of the expected user potential (1.2 Million).

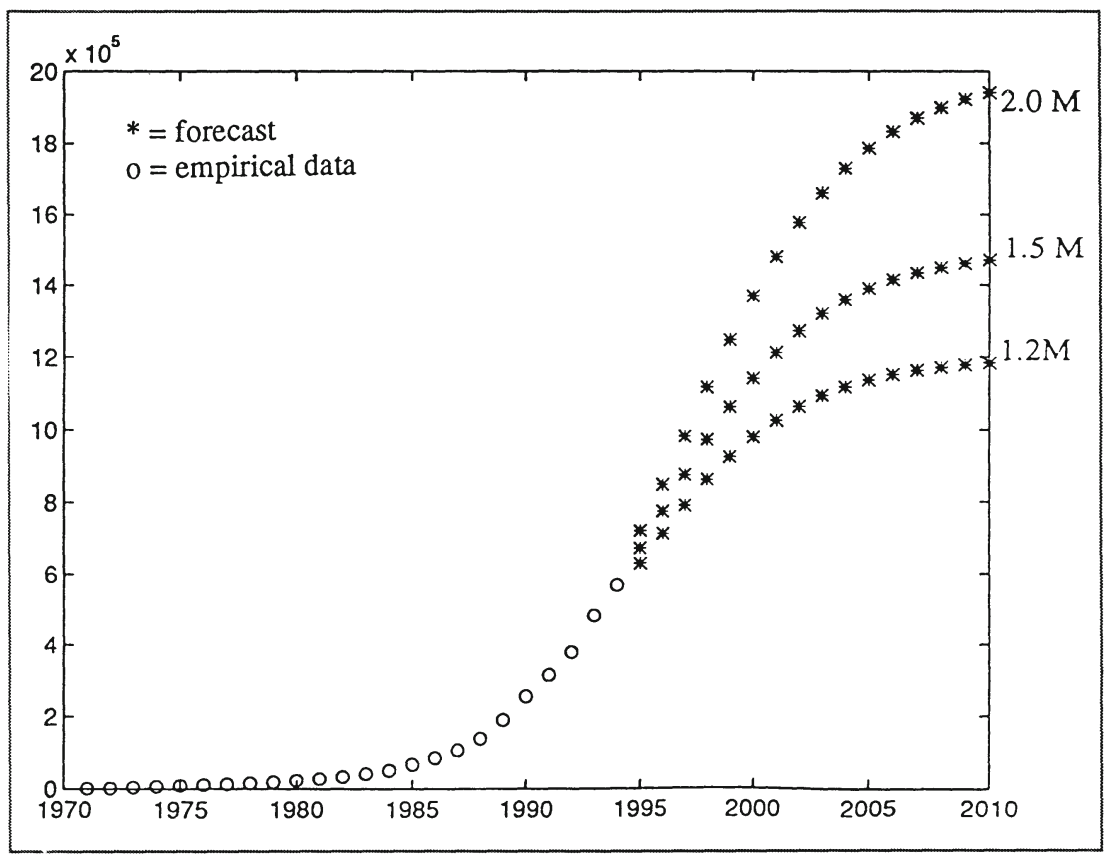

Figure 7 Mobile phone diffusion - empirical data 1971 .. 1994, varying potential

The second part of the analysis tested the forecast sensitivity to expected user potential. The results of the analysis are shown in Figure 7and Table 3. In the analysis the empirical data in the period 1971 .. 1994 was used and the user potential was varied as shown in Table 3.

The user potential variations are based on the EUTELIS (1993) study. The number of licenses at the end of 1994 was 676.000 (in the population of 5 Million the penetration rate was $13.5 \%)$.

Table 3 Parameter values in the analysis

\begin{tabular}{|c|c|c|c|}
\hline$\overline{\mathrm{F}}$ & $\mathrm{F}(1994) / \overline{\mathrm{F}}$ & $\mathrm{C}_{1}$ & $\mathrm{C}_{2}$ \\
\hline \hline $1.2 \mathrm{M}$ & $24 \%$ & -6.86 & 0.29 \\
\hline $1.5 \mathrm{M}$ & $30 \%$ & -7.05 & 0.27 \\
\hline $2.0 \mathrm{M}$ & $40 \%$ & 7.31 & 0.27 \\
\hline $5.0 \mathrm{M}$ & $100 \%$ & -8.18 & 0.26 \\
\hline
\end{tabular}

The tests above point out that the models are very sensitive to the factors that are not pre- 
dictable Each of the sensitivity analysis made above did not lead to the stabilization of model parameter values (in this case because of too short period of empirical data). Stabilization the environment with these unstable parameter values adds unreliability in forecasts. All the models expect the static environment. In the case of mobile phones "static" means fixed telephone and service prices, fixed services available, fixed economy etc. Another point is the user potential: in the models having imitator effect built in the model the driving force is the gap between the number of existing users and the user potential. In the case of mobile phones the user potential is growing as a function of huge amount of factors, among those the ones mentioned above. In fact, the analysis above is based on a compromise between two requirements: length of the time series and exactness built in the data of components. In this case the analysis of component curves is not reliable, however all the component curves have different factors controlling the changes. The effect of these factors may not be joint in the joint curve of its components.

The analysis points out the difficulties in mathematical analysis. The discussion in the beginning of this chapter includes information, that can not be built in the exact models. For an analyst having need to manage the changes of a technology mathematical models are excellent tools to be supplemented by heuristic information available. For exact analysis purposes the models can be used only in very limited applications in the static environment, without rapid changes.

\section{CONCLUSIONS}

For a forecaster there exists a wide variety of mathematical models that can be used in the analysis. The forecaster must be familiar with the properties of the models and especially the restrictions and assumptions in the background. If these details are not taken into account the analysis is unreliable and useless or has negative value to the analyst. This paper has described the background of some mathematical diffusion models - the basic models and some more complicated ones. The purpose of the introduction of the models has not been to explain their properties in detail but to focus on their restrictions and limitations. The existence of tens of basic models and hundreds of variations and still more studies - more or less reliable - in which these models are applied in wide variety of applications does not give facts enough for the beginner analyst to find the right tools for his purposes.

The main findings of this paper are

- the availability of the wide variety of diffusion models and variations of them; it will cause a problem the choice of the right model

- the "unrealistic" assumptions restricting the usability of the models; to be exact there exists no real diffusion process that fills the presumptions of any of the models

- the symmetry - are the diffusion processes really symmetrical

- in most models - especially in the models including advanced flexibility - the parameters of the model do not describe any of the real process; the situation is the same in the case of comparing two processes and in the evaluation of a single process

- in all models there is a need to fix such parameters of the model that will have a revolutionary effect in the results of the analysis

- the model is always a simplified presentation of the real phenomenon; this polarization hides huge amounts of important information that should be known in the management of technological changes

Most of the models expect the knowledge of the potential user population - in some cases we don't know it and in most cases it is changing as a function of time. The behavior outside threshold levels is irregular - how do we really know what is the threshold level. These are the problems of the practical analysis. 
In the world of the exponential growth of information technology the usefulness of the models is still more questionable. The rapid flow of generations and short life cycles of the products do not allow exact mathematical analysis. The models are at their best when they provide interpretable parameters and include the knowledge of the behavior of the process either in its parameters or in the modelling principles itself. This way the analyst learns to understand the behavior of the product in the market and based on this deeper understanding is able to use the information available from the models carefully enough and with the critical grip. A big problem is the misconception of exactness which is caused by the strong belief in mathematical models. The analyst must remember that at its best the models are informative - not exact. Adding the results of mathematical modelling in intuitive knowledge of the process will give the best results. In the literature, the cases analyses are "post ante" analysis giving exact results to show the usefulness of the model. In the real analysis the analyst deals with problems of uncertainty of more parameters that the analysis is able to solve.

\section{REFERENCES}

Ayres, Robert U. (1969) Technological Forecasting and Long-Range Forecasting. McGrawHill Book Company, New York.

Bass, F.M. (1969), A New Product Growth Model for Consumer Durables. Management Science 15, 215-227.

Blackman, A.W. (1972), A Mathematical Model for Trend Forecasts. Technological Forecasting and Social Change 3, 441-452.

Easingwood C., Majahan V., Muller E. (1981), A Non Symmetric Responding Logistic Model for Forecasting Technological Substitution. Technological Forecasting and Social Change 20, 199-213.

Easingwood C., Majahan V., Muller E. (1983), A Nonuniform Influence Innovation Diffusion Model of New Product Acceptance. Marketing Science 2, 273-296.

EUTELIS Consult (1993) Scenario Mobile Communications 2010: Study on Forecast Development and Future Trends in Technical Development and Commercial Provision up to the year 2010. Report to the Commission of the European Communities. CEC Contract No. 48166.

Fichman, R.G. (1992), Information Technology Diffusion: A Review of Empirical Research. In De Gross, Becker, Elam (Eds.), Proc. of the 13th Internatonal Conference on Information Systems, Dallas. pp. 195-206.

Fisher, J.C., Pry, R.H. (1972) A Simple Substitution Model of Technological Change. Technological Forecasting and Social Change 3, 75-78.

Floyd A. (1968), Trend Forecasting: A Methodology for Figures of Merit. In Bright J. (ed.), First Annual Technology and Management Conference. Prentice Hal Inc.

Jaakkola, Hannu (1991) An Analysis of the Diffusion of Information Technology in Finnish Industry. Tampere University of Technology, Publications 70 (In Finnish, English Summary), Tampere.

Jaakkola, Hannu (1991a) Managing By Modelling - How to Manage the Diffusion of a New technology. Proceedings of the Portland International Conference on Management of Engineering and Technology, IEEE.

Jaakkola, Hannu (1992) Modifications and Integration of Diffusion Models. Proceedings of the Joint EURO XII/TIMS XXXI Conference, Helsinki.

Jaakkola, Hannu (1992a) Data Models from Theory to Practice - Modelling the Adoption of New Data Models. In Ohsuga S. (et al.), Information Modelling and Knowledge Bases III, IOS Press, Amsterdam. pp. 675-690.

Jaakkola, Hannu (1992b) The Diffusion of Information Technology in Finnish Industry. In Vuori S., Ylä-Anttila P. (eds.), Mastering Technology Diffusion - The Finnish Experience. ETLA Serie B82-1992, Helsinki. pp. 379-432.

Jaakkola, Hannu (1993) Diffusion Models for Technology Management. In Clark J.D., Troxell 
W.O. (eds.), Design for Competitiveness. Proceedings of the International Conference on Technology Management (April 4-7, 1993), Manufacturing Excellence Centre Press, Denver, Colorado. pp 351-366.

Jaakkola, Hannu (1993a) Toward the Heuristic Model of Technology Diffusion. In the Proceedings of the IFAC World Congress, Preprint Vol. 3, Sydney Australia, International Federation for Automation and Control (IFAC). pp. 441-446.

Jaakkola H., Tenhunen H. (1993b) The Impact of Information Technology on Finnish Industry: A Survey of Two Studies. OECD STI Review 12 (April), pp. 53-80.

Jaakkola, Hannu (1994) The Heuristic Model of Technology Diffusion. In Khalil T.M. et al. (eds.) Management of Technology IV: The Creation of Wealth. Proceedings of the Fourth International Conference on management of Technology (Miami Feb. 27 - March 4, 1994). Industrial Engineering and management Press, Institute of Industrial Engineers, Norcross, Georgia.

Jaakkola, Hannu (1994a), The Heuristic Analysis of the Diffusion. In Management in Transition: Engineering a Changing World. IEEE Proceedings of the IEEE International Engineering Management Conference 1994 (Oct. 17-19, Dayton, Ohio). IEEE, New Jersey, 1994. s. 123-130.

Jaakkola, Hannu (1995), Technology Management with Mathematical Diffusion Models. Working Draft, not published.

Jaakkola, Hannu (1995a), Flexibility in Mathematical Diffusion Models. Working Draft, not published.

Kumar, U., Kumar, V. (1992), Technological Innovation Diffusion: The Proliferation of Substitution Models and Easing the User's Dilemma. IEEE Transactions on Engineering Management 39,2 (May), 158-168.

Lavaraj U.A., Gore A.P. (1990) On Interpreting Probability Distributions Fitted to Times of First Adoption. Technological Forecasting and Social Change 37, 355-370.

Majahan, V., Schoeman, M.E.F. (1977) Generalized Model for the Time Pattern of Diffusion Process. IEEE Transactions on Engineering Management EM-24, 1 (Feb.), 12-18.

Majahan, V., Peterson, R. (1985) Models for Innovation Diffusion. Sage Publications, Beverly Hills.

Majahan, V., Muller, E., Bass, F.M. (1990) New Product Diffusion Models in Marketing: A Review and Directions for Research. Journal of Marketing 54, 1-26.

Majahan V., Muller E., Rajendra K., Srivastva K. (1990a), Determination of Adopter Categories by Using Innovation Diffusion Models. Journal of Marketing Research 27, 37-50.

Mansfield E. (1961), Technical Change and the Rate of Imitation. Econometrica 29 (October), 741-765.

Martino J. (1993), Technological Forecasting for Decision Making. McGraw-Hill Inc.

Martino J. (1994), An Approach to Balancing the Risks f R\&D Performance Goals. In Management in Transition: Engineering a Changing World. IEEE Proceedings of the IEEE International Engineering Management Conference 1994 (Oct. 17-19, Dayton, Ohio). IEEE, New Jersey, 1994. s. 164-167.

Norton J., Bass F. (1987), A Diffusion theory Model of Adoption and Substitution of Successive generations of High Technology Products. Management Science 33, 1069-1086.

Pearl, Raymond (1925), The Biology of Population Growth. Knopf, New York.

Rogers, E. (1983) Diffusion of Innovations. The Free Press, New York.

Sharif M.N., Kabir C.(1976), Systems Dynamics Modelling for Forecasting Multilevel Technological Change. Technological Forecasting and Social Change 8, 353-364.

Sharif, M.N., Ramanathan, K. (1981) Binomial Innovation Diffusion Models with Dynamic Potential Adopter Population. Technological Forecasting and Social Change 20, 63-87.

Skiadas C. (1985), Two Generalized Rational Models for Forecasting Innovation Diffusion. Technological Forecasting and Social Change 27, 39-61.

Telecommunicatons Statistics (1995). Painatuskeskus, Helsinki (in Finnish). 


\section{BIOGRAPHY}

Hannu Jaakkola is a professor of information technology at Tampere University of Technology. He received his Ph.D. (Eng.) degree from Tampere University of Technology in 1991; MSc (computer Science) 1974, BSc (Business Economics) 1979, Licentiate degree (computer science) 1982 in University of Tampere. His research interests cover wide variety of topics in technology management, especially the problems concerning the adoption of new technologies. Additionally he has research activities in the area of software engineering methods and networks. Before present position since 1992 he has been working in different academic positions at Tampere University of Technology, University of Vaasa and University of Tampere. 